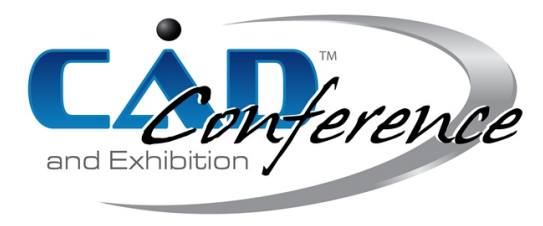

Title:

\title{
Choice of CAD model Adaptation Process for Virtual Reality using Classification Tech- niques
}

Authors:

Florence Danglade, Florence.danglade@ensam.eu, Arts et Metiers ParisTech

Christophe Guillet, Christophe.Guillet@u-bourgogne.fr, Universite de Bourgogne

Keywords:

CAD Model, Virtual Reality, CAD-VR Adaptation, Classification, Machine Learning

DOI: $10.14733 /$ cadconfP.2021.61-65

\section{Introduction:}

Virtual reality and augmented reality (V/AR) techniques are increasingly used in industry ([3], [6]) to support training, design, manufacturing, or maintenance activities. In an industrial context, these V/AR applications integrate 3D models resulting from Computer-Aided Design (CAD) models. Today the adaptation process of a CAD model for a $\mathrm{V} / \mathrm{AR}$ activity is very time-consuming. The adaptation result is often of poor quality or it is poorly adapted to the $\mathrm{V} / \mathrm{AR}$ activity. This is due to a several reasons, CAD and V/AR formats are not compatible; CAD models are too large for V/AR applications; the adaptation process does not meet the need for a specific V/AR activity. All of this slows down the deployment of $\mathrm{V} / \mathrm{AR}$ techniques in the industry ([7], [9]).

However, many tools offer a variety of adaptation functions. An adaptation process applies a succession of these tools and functions. It is difficult to know what the best adaptation process is for a specific V/AR activity. Thus, being able to know a priori the best adaptation process will facilitate the development of $\mathrm{V} / \mathrm{AR}$ applications. Therefore, the development of V/AR application will be shortened. Moreover, the quality of the final $\mathrm{V} / \mathrm{AR}$ application will be improved. We propose to help the developer of the $\mathrm{V} / \mathrm{AR}$ application to choose the best CAD model adaptation process. In previous work ([4], [5]), it was proposed a method to evaluate an adaptation process of a CAD model by using machine learning techniques in a different context. In this paper, we propose a new approach to predict the best adaptation process of CAD models for a specific $\mathrm{V} / \mathrm{AR}$ application using techniques of classification. One challenge was to model a specific adaptation process of CAD data for each objective of $\mathrm{V} / \mathrm{AR}$ application. A second challenge was to identify variables that influence the choice of adaptation process. The proposed approach will be illustrated and validated on examples of adaptation for various original CAD models and industrial V/AR applications.

Method to Choose a CAD Model Adaptation Process for V/AR using Classification techniques :

Overall approach. Our overall approach is based on four main steps (Fig. 1). First step consists in formalizing the requirements related to the $\mathrm{V} / \mathrm{AR}$ activities. Then, a learning database is built by describing the requirements, the original CAD models, and the adaptation processes. Learning data are compiled in a matrix (Tab. 1) where each row represent a case that describes a known case of CAD model adaptation for a specific $\mathrm{V} / \mathrm{AR}$ activity. Columns of the matrix represent vectors of variables that describe CAD 


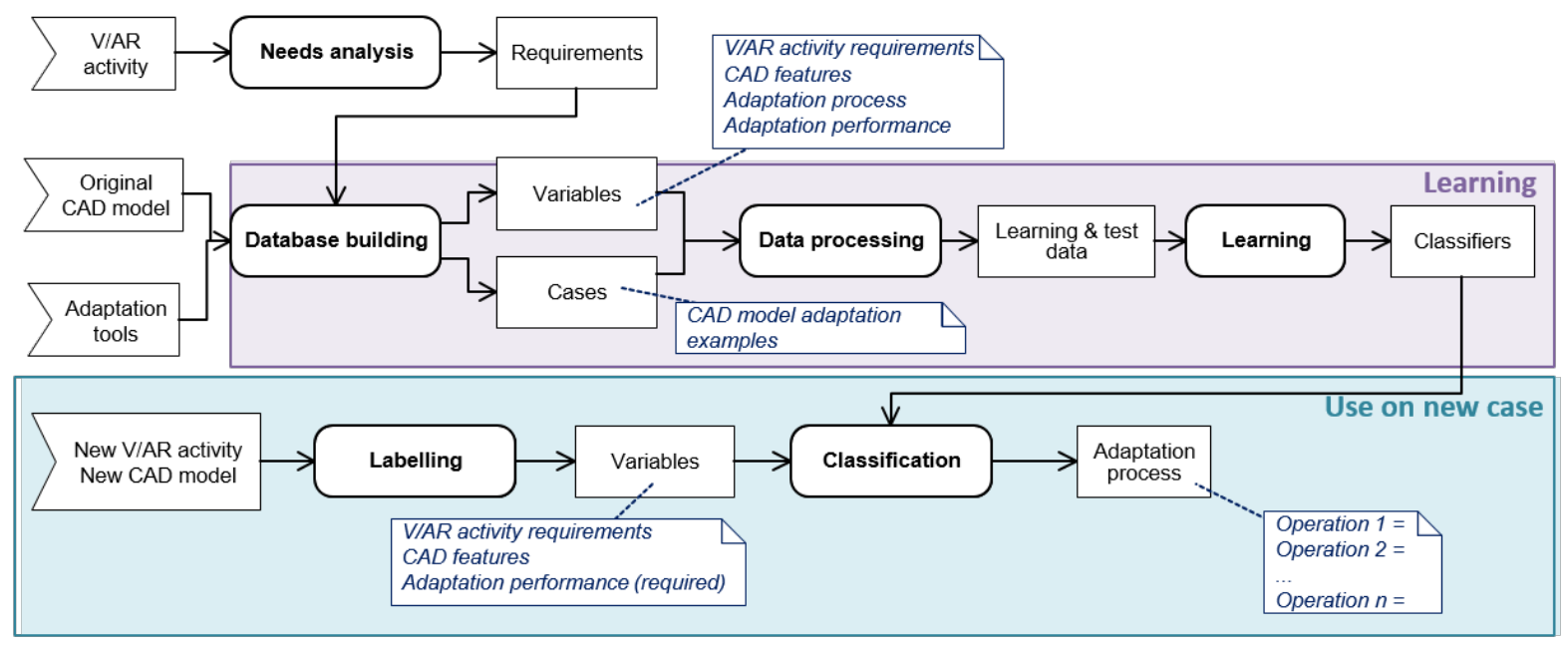

Fig. 1: Framework to choose a CAD model adaptation process for $\mathrm{V} / \mathrm{AR}$ using classification techniques.

\begin{tabular}{|c|c|c|c|c|c|c|}
\hline Variables: & $\begin{array}{l}\mathrm{V} / \mathrm{AR} \\
\text { Objectives }\end{array}$ & $\begin{array}{l}\text { Quality } \\
\text { Criteria }\end{array}$ & $\begin{array}{l}\text { Satisfaction } \\
\text { Levels }\end{array}$ & $\begin{array}{l}\text { CAD } \\
\text { Features }\end{array}$ & $\begin{array}{l}\text { Adaptation } \\
\text { Operations }\end{array}$ & $\begin{array}{l}\text { Adaption } \\
\text { Time }\end{array}$ \\
\hline Values: & Qualitative & Numeric & Numeric & Numeric & Qualitative & Numeric \\
\hline $\begin{array}{l}\text { Known } \\
\text { case 1: }\end{array}$ & $X \operatorname{var}(1)$ & $\begin{array}{l}\text { Xreq, 1(1): } \\
\text { Xreq, 6(1) }\end{array}$ & $\begin{array}{l}X q, 1(1): \\
X q, 6(1) \\
X s q(1)\end{array}$ & $X c a d, j(1)$ & $\begin{array}{l}\text { Yop, } 0(1): \\
\text { Yop, } 5(1)\end{array}$ & $\begin{array}{l}Y \operatorname{tim}(1), \\
X s t(1)\end{array}$ \\
\hline $\begin{array}{l}\text { New } \\
\text { case a: }\end{array}$ & $X \operatorname{var}(a)$ & $\begin{array}{l}\text { Xreq, } 1(a): \\
\text { Xreq, } 6(a)\end{array}$ & $\begin{array}{l}X q, 1(a)=1: \\
X q, 6(a)=1 \\
X s q(a)=1\end{array}$ & $X c a d, j(a)$ & $\begin{array}{l}Y o p, 0(a)=?: \\
Y o p, 5(a)=?\end{array}$ & $\begin{array}{l}Y \operatorname{tim}(a)=?, \\
X \operatorname{st}(a)=1\end{array}$ \\
\hline
\end{tabular}

Table 1: Matrix for learning (known cases) and prediction (new cases) database.

models, requirements, and processes. In a third step, a set of tools for data processing is applied (e.g. normalization or reduction). This processing will allow to adapt the data to the learning algorithms. The next learning step consists in configuring classifiers for the choice of adaptation operations. In the end, for a new case (new CAD model or new V/AR activity), the configured classifiers propose a set of adaptation operations.

Need analysis. In order to meet the needs of the application user, the 3D model must be specifically adapted to the purpose of the activity. It is therefore essential to clearly identify the specifications for each type of activity. These requirements are described using four variables (Tab. 1: Xvar, Xreq,, $X q, i$ and $X s q$. Xvar is a qualitative variable that describes the objective by specifying: i) the type of application (AR or VR), ii) the product life phase (design, manufacturing, maintenance, or service), iii) the type of activity (visualization, review, assistance, or training). Xreq, $i$ is a vector of six variables that represents the expected level of quality criteria. Xreq, $i$ takes the value of 0 if the requirement is low and 1 if it is high. Tab. 2 gives the criteria and requirements for low and high levels. $X q, i$ is a vector of six variables that gives the satisfaction level of the adapted 3D model for each criterion $X r e q, i$. The variable $X q, i$ is 0 if the result doesn't meet the criterion; $X q, i$ is 0.5 if the result partially meets the criterion; $X q, i$ is 1 if the result fully meets the criterion; $X q, i$ is 1.5 in case of over-quality. The global 


\begin{tabular}{l|lll} 
Requirements: & Weight & $\begin{array}{l}\text { Low or no requirements } \\
(X r e q, i=0)\end{array}$ & $\begin{array}{l}\text { Very high requirements } \\
(X r e q, i=1)\end{array}$ \\
\hline $\begin{array}{l}\text { Xreq, } 1: \\
\text { visualizations quality }\end{array}$ & $w 1=1$ & No visualization requirements & $\begin{array}{l}\text { No latency or discomfort during } \\
\text { visualization }\end{array}$ \\
\hline $\begin{array}{l}\text { Xreq, } 2: \\
\text { shapes, dimensions } \\
\text { precision }\end{array}$ & $w 2=0.8$ & $\begin{array}{l}\text { The adapted and original } \\
\text { CAD model may differ }\end{array}$ & $\begin{array}{l}\text { The adapted model is completely } \\
\text { identical to the original CAD } \\
\text { model }\end{array}$ \\
\hline $\begin{array}{l}\text { Xreq, } 3: \\
\text { level of details and } \\
\text { realism }\end{array}$ & $w 3=0.8$ & $\begin{array}{l}\text { A schematic representation is } \\
\text { sufficient }\end{array}$ & $\begin{array}{l}\text { The adapted model has the same } \\
\text { rendering as the physical product }\end{array}$ \\
\hline $\begin{array}{l}X r e q, 4: \\
\text { physics preservation }\end{array}$ & $w 4=0.6$ & No physics requirements & $\begin{array}{l}\text { The adapted model behavior is } \\
\text { the same as the physical product }\end{array}$ \\
\hline $\begin{array}{l}X r e q, 5: \\
\text { changes facility }\end{array}$ & $w 5=0.5$ & No change is required & $\begin{array}{l}\text { Real-time changes on adapted } \\
\text { model are possible }\end{array}$ \\
\hline $\begin{array}{l}X r e q, 6: \\
\text { collaborative } \\
\text { work }\end{array}$ & $w 6=0.5$ & $\begin{array}{l}\text { No collaborative work is re- } \\
\text { quired }\end{array}$ & $\begin{array}{l}\text { All collaborative works are re- } \\
\text { quired }\end{array}$
\end{tabular}

Table 2: Criteria of quality requirements of the 3D model for a V/AR activity.

level of satisfaction $X s q$ is evaluated from the weighted average of $X q, i$ values by factors $w i$.

Database building. There are three main families of adaptation tools: CAD software, adaptation software, and computer graphic software. Each tool offers one or more adaptation functions: components removing, details removing, local simplification, global simplification, or idealization. It is often necessary to use several adaptation tools simultaneously. We end up with a large number of possible processes. An adaptation process will be described by a sequence of $n$ operations. The variables $Y$ op, $n$ describe operations by specifying the used tool and adaptation function. The values of variables $X s q$ (level of overall satisfaction) and $Y$ st (adaptation duration satisfaction) indicate the adaptation performance. Today, choosing an adaptation process is often an arbitrary decision. The performance of the adaptation is therefore unsatisfactory. The objective of this work is to predict the output variables Yop,n and Ytim (adaptation duration) with satisfactions $X s q$ and $Y$ st closed to 1.

When the designer of $\mathrm{V} / \mathrm{AR}$ application prepares a CAD model, he uses subjective criteria to choose his adaptation operations. These criteria are related to the size of the CAD model (e.g. area, volume), its shape (e.g. compactness, curvatures, number of faces, number of details, types of faces), and product features (e.g. number of similar components in the product, links with other components). These criteria should be formalized to be a potential variable for learning and classification. Thus, the database initially contains a set of $34 X C A D, j$ variables, $j$ is the index of the variable. These variables were first chosen to best characterize a CAD model, ensuring that the values of variables are not the same for two different CAD models.

Data processing. First, this step consists in applying operations of normalization and repair of missing or aberrant data. Then variables that do not influence the choice of the adaptation process are eliminated. Principal Component Analysis give projections of variables on factorial plans. These plans and correlation matrix makes it possible to identify the correlated variables with each other and their correlation coefficient. Thus, these methods allow to identify among the $34 \mathrm{XCAD}, \mathrm{j}$ variables, the most correlated variables with the $Y o p, n, X s q, X s t$ and $Y$ tim variables. The least correlated variables will 


\begin{tabular}{r|cccccc} 
Prediction errors : & $Y o p, 0$ & Yop,1 & Yop, 2 & Yop, 3 & Yop,4 & Yop, 5 \\
\hline Total: & $3.20 \%$ & $16.60 \%$ & $9.10 \%$ & $7.50 \%$ & $35 \%$ & $42 \%$ \\
\hline Giving an over quality: & $3.20 \%$ & $5.80 \%$ & $3.30 \%$ & $4.20 \%$ & $31 \%$ & $23 \%$ \\
\hline Reducing the quality: & $0 \%$ & $10.80 \%$ & $5.80 \%$ & $3.30 \%$ & $4 \%$ & $19 \%$
\end{tabular}

Table 3: Predictions errors of SVM classifier.

be removed. This will improve the performance of the classification and facilitate future data setting.

Learning. This step consists in selecting and configuring classifiers to predict the best operation sequence for a new CAD model or a new V/AR objective. Data are partitioned into a group for learning ( $80 \%$ of cases) and into a group for tests $(20 \%$ of cases). The total number of cases is not very high. The $\mathrm{k}$-fold cross-validation method (with $\mathrm{k}=10$ ) improves the classification performance. Various classifiers are tested and then optimized: Decision Tree, Artificial Neural Network, and Support Vector Machines. Classifiers are evaluated with the group of cases for tests using criteria of prediction errors and F1 scores. Among the prediction errors, we will distinguish the results for which the performance of the adaptation is degraded and those that lead to an over-quality.

Classification on a new case. For a new case of CAD model or V/AR activity, known variables (Tab. 1) are labeled. The global satisfaction levels $X s q L$ and $Y s t$ are set to 1. The configured classifiers predict the Yop, $n$ and Ytim output variables. The proposed adaptation operations are optimal without compromise. Therefore, the developer of the V/AR application can follow the proposed process.

$\underline{\text { Results : }}$

We evaluated the proposed method on eight different applications of $\mathrm{V} / \mathrm{AR}$. Tools for adaptation are CATIAV5 [2] (CAD software), Pixyz [8] (adaptation software), and 3DSMAX [1] (computer graphic software). The learning database contains 600 cases of adapted CAD models. Principal component analysis and correlation matrix allowed the reduction of the Xcad,j variables number from 34 to 6 variables: number of faces, compacity, box volume, ratio of volumes between part and product, ratio of areas, number of parts. These variables are not correlated with each other, and they are correctly correlated with the variables $X q, i, X s q, Y o p, n, X s t$, and Ytime. The best classifier is a Support Vector Machines algorithm with a sigmoid kernel. The Tab. 3 shows acceptable prediction errors and high F1 scores for this classifier. The addition of new cases in the database should improve these scores.

The Fig. 2 gives examples of the use of our method for two parts and two V/AR activities. Regarding the part 1 without prediction (Fig. 2 (a)), the designer of V/AR application re-models the part and keeps all the details as a precaution. This leads to an over quality and a waste of time. The predicted adaptation process for the objective of AR guidance (Fig. 2 (b)), consists in transforming the CAD model into a $2 \mathrm{D}$ surface by idealisation, keeping all the details. Lighter, the model is no longer over-quality. For the objective of VR training (Fig. 2 (c)), the predicted adaptation process consists in removing all details. The result is completely satisfactory for the intended objective and the time saving is significant. Regarding the part 2, the designer of the $\mathrm{V} / \mathrm{AR}$ application simplifies the sub-assembly by first removing small details and then reducing the number of polygons. The result is unsatisfactory because the requirements are not taken into account (conservation of details like screws for AR guidance and precision of shapes for VR training). On the other hand, these requirements are well respected for models with predicted adaptation processes. In summary, for these examples, the prediction of the adaptation process provides an adaptation with a level of quality that perfectly meets the need.

Proceedings of CAD'21, Barcelona, Spain, July 5-7, 2021, 61-65

(C) 2021 CAD Solutions, LLC, http://www.cad-conference.net 


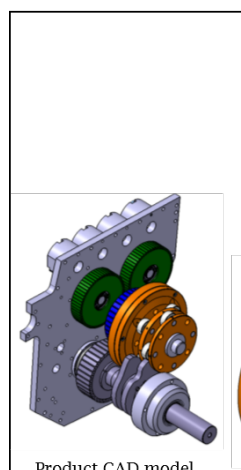

Product CAD model (original)
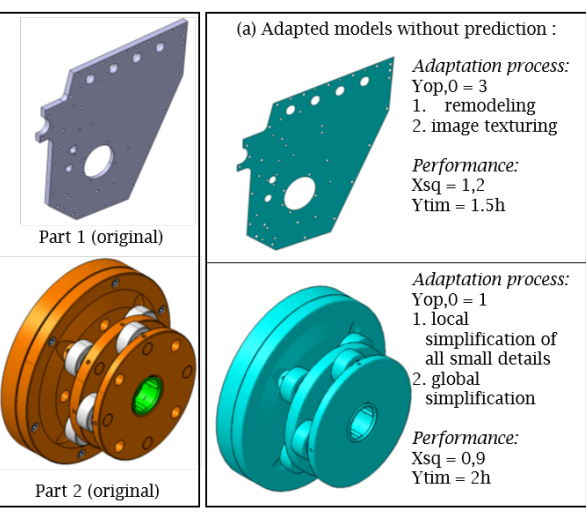
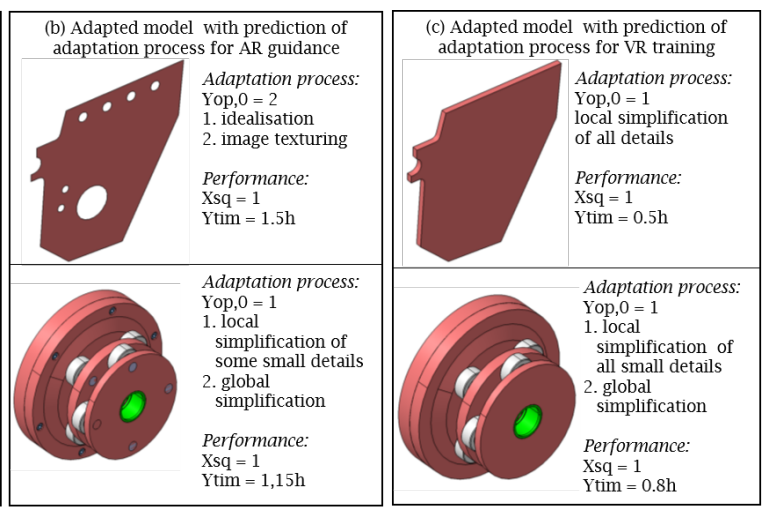

Fig. 2: Example of use on new case.(a) without prediction. (b),(c) with prediction of adaptation process.

\section{Conclusions:}

In this paper, a classification method for the choice of a CAD model adaptation process for virtual reality had been developed. While the designer relies on subjective criteria to choose the adaptation operations, our method allowed to predict the best process from selected variables. The results obtained on new cases are promising. This method helps the developer to adapt the CAD model with an ideal level of satisfaction (quality and time) by taking account the specific needs of the V/AR activity. In addition to ensuring a good quality of the adapted model, the proposed method makes it possible to know a priori the adaptation process without having to test it beforehand in the final application of $\mathrm{V} / \mathrm{AR}$. This avoids long iterations between CAD software, adaptation tools, and V/AR application creation tools. In the end, this should facilitate the deployment of $\mathrm{V} / \mathrm{AR}$ techniques in the industry. With our method, the process of operations is predicted before operations are carried out. In future work, we could predict the $\mathrm{n}+1$ operation in real-time by taking into account the actual result of the $\mathrm{n}$ operation.

\section{References:}

[1] 3DS MAX, https://www .autodesk.fr/products/3ds-max/overview.

[2] CATIA, http://www.3ds.com/products-services/catia/.

[3] Damiani, L. ; Demartini, M. ; Guizzi, G.; Revetria, R. ;Tonelli, F.: Augmented and virtual reality applications in industrial systems: a qualitative review towards the industry 4.0 era, IFACPapersOnLine, 51, 624-630. https://doi.org/10.1016/j.ifacol.2018.08.388

[4] Danglade, F. ; Veron, P. ; Pernot, JP. ; Fine, L. : Estimation of CAD model simplification impact on CFD analysis using machine learning techniques, CAD COnference, 2015.

[5] Danglade, F. ;Pernot, JP. ; Veron, P. ; Fine, L. : A priori evaluation of simulation models preparation processes using artificial intelligence techniques, Computers in Industry, 91, 2017, 45-61. https://doi.org/10.1016/j.compind.2017.06.001

[6] Dini, G. ; Dalle Mura, M. : Application of Augmented Reality Techniques in Through-life Engineering Services, Procedia CIRP, 38, 2015, 14-23. https://doi.org/10.1016/j.procir.2015.07.044

[7] Horejsi, P. : Augmented Reality System for Virtual Training of Parts Assembly, Procedia Engineering, 100, 2015, 699-706. https://doi.org/10.1016/j.proeng.2015.01.422

[8] Pixyz, https://www.pixyz-software.com.

[9] Wolfartsberger, J. : Analyzing the potential of Virtual Reality for engineering design review, Automation in Construction, 104, 2019, 27-37. https://doi.org/10.1016/j.autcon.2019.03.018 